\title{
Materials Selection in Micromechanical Design: An Application of the Ashby Approach
}

\author{
V. T. Srikar and S. Mark Spearing, Member, ASME
}

\begin{abstract}
The set of materials available to microsystems designers is rapidly expanding. Techniques now exist to introduce and integrate a large number of metals, alloys, ceramics, glasses, polymers, and elastomers into microsystems, motivating the need for a rational approach for materials selection in microsystems design. As a step toward such an approach, we focus on the initial stages of materials selection for micromechanical structures with minimum feature sizes greater than $1 \mu \mathrm{m}$. The variation of mechanical properties with length scale and processing parameters is discussed. Bounds for initial design values of several properties are suggested and the necessity for the measurement of other properties (especially residual stresses and intrinsic loss coefficients) is discussed. Adapting the methods pioneered by Ashby et al., materials indices are formulated for a number of properties and materials selection charts are presented. These concepts are applied to illustrate initial materials selection for shock-resistant microbeams, force sensors, micromechanical filters, and micromachined flexures. Issues associated with the integration of materials into microsystems are briefly discussed.

[878]
\end{abstract}

Index Terms-Design, materials selection, MEMS, sensors.

\section{INTRODUCTION}

I $\mathrm{N}$ RECENT years, enormous progress has been made in the design, fabrication, and commercialization of microsystems (also known as microelectromechanical systems (MEMS)) [1], [2]. Associated with these advances is an expansion of the set of materials available to MEMS designers. In addition to the materials traditionally used in silicon microelectronics (singlecrystal and polycrystalline silicon, silicon oxide, silicon nitride, and aluminum, for instance), it is now possible to introduce and integrate a wide variety of metals and alloys (ex: $\mathrm{Cu}, \mathrm{Ni}$, $\mathrm{Ni}-\mathrm{Fe}, \mathrm{Ni}-\mathrm{Ti}, \mathrm{Au}, \mathrm{Pd}$, and $\mathrm{W}$ ), ceramics and glasses (ex: $\mathrm{SiC}$, $\mathrm{ZrO}_{2}, \mathrm{CeO}_{2}$, quartz, $\mathrm{ZnO}$, lead zirconium titanate (PZT), and diamond) and polymers and elastomers (ex: polyimide and SU8) into microsystems [3]. This capability motivates the following question: given a design concept, how is a designer to choose a material (or set of materials) to optimize performance and reliability?

An early attempt at an answer was made by MacDonald et al. [4], who identified three requirements for materials to be used in MEMS: compatibility with silicon technology, desirable electromechanical properties, and low values of residual stresses. More recently, Spearing surveyed materials issues in

Manuscript received May 22, 2002; revised September 20, 2002. Subject Editor W. N. Sharpe, Jr.

The authors are with the Department of Aeronautics and Astronautics, Massachusetts Institute of Technology, Cambridge, MA 02139 USA. (email vts@alum.mit.edu; srikar@mtl.mit.edu; spearing@mit.edu)

Digital Object Identifier 10.1109/JMEMS.2002.807466
MEMS and observed that the approach commonly used for the selection of materials in macroscale design (hereafter called the Ashby approach after one of the pioneers in that field [7]-[14]) can also be applied to microsystems, and discussed the selection of materials for gyroscopes, pressure sensors, and microturbines [5], [6]. In this paper, we expand upon this observation and discuss in detail the applicability of the Ashby method to microsystems. Specifically, we discuss the dependence of mechanical properties on length scale and processing parameters, the formulation of materials indices, and the use of materials selection charts. These concepts are then applied to illustrate the selection of materials for shock resistant microbeams, force sensors, micromechanical filters, and micromachined flexures.

This paper is organized as follows. Section II considers the materials used in the fabrication of microsystems. The mechanical and structural properties of interest are discussed in Section III and Section IV, respectively. The design process, and the need for mechanical property measurements at different stages, is discussed in Section V. The Ashby methodology (including the concepts of material indexes and material properties charts) is reviewed in Section VI. The application of this methodology to microsystems design is illustrated in Section VII with the aid of several case studies. Areas for future research, including issues associated with the integration of materials into microsystems, are briefly discussed in Section VIII.

\section{MATERIALS FOR MicRosystems}

By a combination of various processing techniques such as bulk-micromachining, surface-micromachining, LIGA (electroplating into molds), and soft lithography, it is now possible to introduce, shape, and integrate a large number of engineering materials into micromechanical elements [3]. These materials are traditionally grouped into four classes: metals and alloys, glasses and ceramics, polymers and elastomers, and composites (combination of materials from different classes). Examples of the members of these classes were listed earlier. In the context of MEMS, Spearing suggested a complimentary classification: structural materials are those that constitute load-bearing beams, plates, membranes and other mechanical elements, and transducer materials constitute devices that convert information and energy from one physical domain into another [5].

Many bulk materials are commercially available in standard form with specified property values. Microscale materials are not. With very few exceptions, mechanical property measurements are invariably required, at some stage, in the design of microsystems [15]. 


\section{PROPERTIES OF MATERIALS}

The properties of materials commonly required in mechanical design are the Young's modulus $(\mathrm{E})$, density $(\rho)$, Poisson's ratio $(\nu)$, fracture strength $\left(\sigma_{\mathrm{F}}\right)$, yield strength $\left(\sigma_{\mathrm{Y}}\right)$, fracture toughness $\left(\mathrm{K}_{\mathrm{IC}}\right)$, coefficient of thermal expansion $(\alpha)$, specific heat per unit mass $\left(\mathrm{C}_{\mathrm{P}}\right)$, loss coefficient $(\eta)$, and residual stress $\left(\sigma_{\mathrm{R}}\right)$ [9]. [Strictly speaking, the fracture strength is the property of a structure, whereas the fracture toughness is a materials property. In order to use fracture toughness values in microsystems design, however, it is necessary to evaluate flaw sizes in microscale structures using nondestructive techniques. This is extremely hard. Therefore, the tensile fracture strengths of representative structures (or the probabilistic distribution of tensile fracture strengths) are commonly used as design metrics.]

Compared to the properties of macroscale ("bulk") structures, the properties of microscale structures can potentially be functions of the length scale as well as the details of the processing techniques employed. However, by focusing on structures with minimum feature sizes greater than $1 \mu \mathrm{m}$, it is possible to quantitatively relate micromechanical properties to bulk properties in many cases.

Properties whose physical origins lie at the atomic scale (size and weight of atoms, nature of bonding and bond density, etc.) are, to first order, expected to be the same in micromechanical $(>1 \mu \mathrm{m})$ and bulk structures. These properties include the Young's modulus, density, Poisson's ratio, coefficient of linear expansion, and the specific heat per unit mass. This expectation can be confirmed in the case of the Young's modulus, which has been measured in a number of microscale materials. Sharpe has tabulated initial design values based on an extensive survey of such measurements [16]. These values are listed in Table I along with nominal bulk values tabulated by Ashby and Jones [9]. We conclude that $0.8 \mathrm{E}_{\text {bulk }}<\mathrm{E}_{\mu}<\mathrm{E}_{\text {bulk }}$, where the subscript ' $\mu$ ' indicates 'microscale.' Therefore, for the initial stages of micromechanical design, bulk values of these properties, which are often easily available in handbooks, can be used.

Other properties, such as the fracture and yield strengths, loss coefficient, and residual stress, have values determined by interactions at multiple length scales (typically in the range 0.01 to $1 \mu \mathrm{m})$ and can vary dramatically in microscale structures compared to bulk values. In general, strength values increase as the length scale decreases due to the reduced probability of occurrence of critical defects [5] and due to increased constraints for dislocation motion. In particular, the yield strengths of thin metal films can exceed bulk values by upto an order of magnitude [17]. Therefore, bulk values can be used as conservative limits for the yield and fracture strength.

Based on this, and on the following discussion of intrinsic loss coefficients and residual stresses, Table II summarizes the initial design values for various material properties.

\section{A. Intrinsic Loss Coefficient $\left(\eta_{i}\right)$}

The loss coefficient $(\eta)$ is a measure of the energy dissipated during vibration of micromechanical resonators and oscillators.
TABLE I

COMPARISON OF BULK AND MiCROSCALE Properties

\begin{tabular}{l|l|l|l|l}
\hline & $\begin{array}{l}\mathrm{E}_{\text {bulk }} \\
(\mathrm{GPa}) \\
\text { Ref. 9 }\end{array}$ & $\begin{array}{l}\mathrm{E}_{\mu} \\
(\mathrm{GPa}) \\
\text { Ref. 16 }\end{array}$ & $\begin{array}{l}\sigma_{\mathrm{F}, \text { bulk }} \\
(\mathrm{MPa}) \\
\text { Ref. 9 }\end{array}$ & $\begin{array}{l}\sigma_{\mathrm{F}, \mu} \\
(\mathrm{MPa}) \\
\text { Ref. 16 }\end{array}$ \\
\hline Aluminum & 69 & 70 & 200 & 150 \\
\hline Copper & 124 & 120 & 400 & 350 \\
\hline Gold & 82 & 70 & 220 & 300 \\
\hline Nickel & 214 & 180 & 400 & 500 \\
\hline Ni-Fe alloy & $130-$ & 120 & $400-$ & 1600 \\
& 234 & & 2000 & \\
\hline $\begin{array}{l}\text { Diamond-like } \\
\text { Carbon }\end{array}$ & $700-$ & 800 & $\begin{array}{l}8000- \\
10000\end{array}$ & 8000 \\
\hline Polysilicon & $130-$ & 160 & $2000-$ & $1200-$ \\
& 180 & & 4000 & 3000 \\
\hline $\begin{array}{l}\text { Single-crystal } \\
\text { Silicon }\end{array}$ & $130-$ & $125-$ & $2000-$ & $>1000$ \\
180 & 180 & 4000 & \\
\hline $\begin{array}{l}\text { Silicon } \\
\text { carbide }\end{array}$ & $430-$ & 400 & $4000-$ & - \\
\hline $\begin{array}{l}\text { Silicon } \\
\text { nitride }\end{array}$ & $280-$ & 250 & $\begin{array}{l}5000- \\
8000\end{array}$ & 6000 \\
\hline Silicon oxide & $50-80$ & 70 & $800-$ & 1000 \\
& 50 & & 1100 & \\
\hline
\end{tabular}

TABLE II

RECOMMENDED INITIAL DESIGN VALUES OF MATERIAL PROPERTIES

\begin{tabular}{l|l}
\hline Property & Recommendation \\
\hline Density, $\rho\left[\mathrm{kg} \mathrm{m}^{-3}\right]$ & $\rho_{\mu} \approx \rho_{\text {bulk }}$ \\
\hline $\begin{array}{l}\text { Young's modulus, } \mathrm{E} \\
{[\mathrm{GPa}]}\end{array}$ & $0.8 \mathrm{E}_{\text {bulk }} \leq \mathrm{E}_{\mu} \leq \mathrm{E}_{\text {bulk }}$ \\
\hline $\begin{array}{l}\text { Poisson's ratio, } \nu[-] \\
\text { Fracture strength, } \sigma_{\mathrm{F}} \\
{[\mathrm{MPa}]}\end{array}$ & $\sigma_{F, \mu} \approx \sigma_{F, \text { bulk }}$ \\
\hline $\begin{array}{l}\text { Linear expansion } \\
\text { coefficient, } \alpha\left[\mathrm{K}^{-1}\right]\end{array}$ & $\alpha_{\mu} \approx \alpha_{b u l k}$ \\
\hline $\begin{array}{l}\text { Specific heat, } \mathrm{C}_{\mathrm{p}} \\
{\left[\mathrm{J} \mathrm{Kg} \mathrm{K}^{-1}\right]}\end{array}$ & $C_{P, \mu} \approx C_{P, b u l k}$ \\
\hline $\begin{array}{l}\text { Intrinsic loss } \\
\text { Coefficient, } \eta_{\mathrm{i}}[-]\end{array}$ & $10^{-2}<\eta_{i} \quad$ (polymers) \\
& $10^{-5}<\eta_{i}<10^{-3}$ (metals) \\
\hline $\begin{array}{l}\text { Residual stress, } \sigma_{\mathrm{R}} \\
{[\mathrm{MPa}]}\end{array}$ & $-1 \mathrm{GPa}<\sigma_{\mathrm{R}}<10^{-4}$ (ceramics) \\
\hline
\end{tabular}

In the MEMS literature, this quantity is usually reported as the inverse of the mechanical quality factor $\left(\mathrm{Q}^{-1}\right)$. For small values of $\eta(\eta<0.01)$, the two quantities are related simply by [7]

$$
\eta=Q^{-1}=\frac{\Delta W}{2 \pi W}
$$


where $\Delta \mathrm{W}$ is the energy dissipated per cycle of vibration and $\mathrm{W}$ is the maximum stored energy. The total damping can be separated into two components given by

$$
\eta=\eta_{e}+\eta_{i}
$$

where the subscripts e and i denote extrinsic and intrinsic, respectively. The primary sources of extrinsic losses include air-damping, squeezed-film damping, and acoustic losses through the supports of the resonators [18]. Under ambient conditions, these dominate in microscale devices and limit the loss coefficient to values in the range 0.01 to 0.1 . If extrinsic losses are minimized, by operating the resonator in vacuum, for example, then intrinsic losses (thermoelastic damping, damping due to dislocations and grain boundary sliding, etc.) determine the magnitude of the loss coefficient. The value of $\eta_{\mathrm{i}}$ is a function of several material properties $\left(\mathrm{E}, \rho, \mathrm{C}_{\mathrm{p}}\right)$, density and distribution of crystallographic defects, morphology and size of grains, and the amplitude and frequency of vibration [19]-[21]. It is not possible at present to predict the value of $\eta_{i}$ for any specified combination of processes and materials. However, based on measurements in macroscale and microscale resonators [19]-[29], we can suggest bounds for initial design values at $300 \mathrm{~K}$ :

$$
\begin{aligned}
\eta_{i} & >10^{-2}(\text { polymers }) \\
10^{-3} & >\eta_{i}>10^{-5} \text { (metals) } \\
10^{-4} & >\eta_{i}>10^{-7} \text { (ceramics). }
\end{aligned}
$$

\section{B. Intrinsic Residual Stress}

In addition to stresses generated by applied thermomechanical loads, intrinsic stresses arising from the details of the growth process contribute significantly to residual stresses in micromachined structures [1], [3], [30]. Specifically, intrinsic stresses in deposited thin films can vary from $1 \mathrm{GPa}$ in compression to $1 \mathrm{GPa}$ in tension. Moreover, relatively small variations in process parameters (argon pressure in sputtering [30], substrate temperature in evaporation [31], [32], and annealing temperature in chemical-vapor deposition [3], for example) can lead to changes of several hundred megapascals in the intrinsic stress. The mechanisms responsible for the origin and evolution of such stresses in thin films are a topic of considerable current research [31] and it is not possible at present to predict the magnitudes of intrinsic stresses for any specified combinations of processes and materials. Therefore, measurements of residual stress are invariably necessary even in the initial stages of microsystems design [15].

It is worth noting that some bulk micromachining processes, where the structure is sculpted from a stress-free starting material, can yield micromechanical devices with essentially zero residual stress.

\section{PROPERTIES OF StruCtURES}

Microfabrication techniques typically yield structures that are extrusions of two-dimensional shapes. Uniform rectangular cross sections dominate micromechanical design. As a

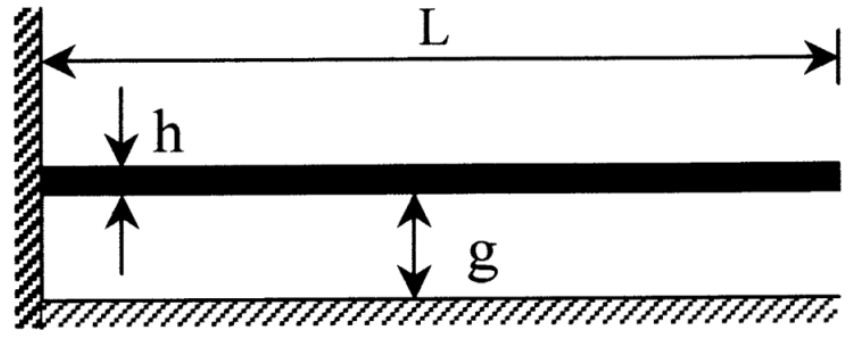

Fig. 1. Schematic illustration of a cantilever microbeam of length $L$ and thickness $\mathrm{h}$ suspended over a fixed substrate.

representative structure, consider a cantilever beam element (see Fig. 1) of length L, thickness h, width b, and suspended over a fixed substrate. The goal of this section is to formulate materials indices $\left(M_{i}\right)$ for various properties of this structure. As shown below, these indices capture the dependence of structural characteristics on material properties.

The mass $(\mathrm{m})$ of the beam is simply

$$
m=(L b h)(\rho) \quad M_{1}=\rho
$$

The sole material property in (4) is the density; this is expressed as a materials index, $\mathrm{M}_{1}$.

Inertial loads $(\mathrm{P})$ induced by applying pulses of acceleration (a) are given by

$$
P=m a=(L b h)(\rho)(a) \quad M_{2}=\rho .
$$

The bending stiffness (S) is defined as the ratio of the applied bending load $(\mathrm{P})$ to the deflection of the beam $(\delta)$ and is given by [7]

$$
S=\frac{P}{\delta}=\left(\frac{b h^{3}}{4 L^{3}}\right)(E) \quad M_{3}=E
$$

The tip deflection is related to the maximum tensile stress $(\sigma)$ in the beam through the expression [7]

$$
\delta=\left(\frac{2 L^{2}}{3 h}\right)\left(\frac{\sigma}{E}\right) \quad M_{4}=\frac{\sigma_{F}}{E} .
$$

The frequency of bending vibrations of the beam is expressed as [7]

$$
f=\left(\frac{0.16 h}{L^{2}}\right) \sqrt{\frac{E}{\rho}} \quad M_{5}=\sqrt{\frac{E}{\rho}} .
$$

The materials index $M_{5}$ is identically equal to the speed of longitudinal elastic waves in the material.

In presenting the above equations, we have implicitly assumed that the equations of continuum mechanics are applicable to structures with feature sizes $>1 \mu \mathrm{m}$. Experience indicates that this assumption is generally valid [1].

\section{Design of Microsystems}

A microsystem is a collection of interconnected subsystems enclosed in a package (see Fig. 2). The package mediates the exchange of information (signals), matter, and energy with the surrounding environment. Depending on their primary function, the subsystems can be identified as micromechanical, 


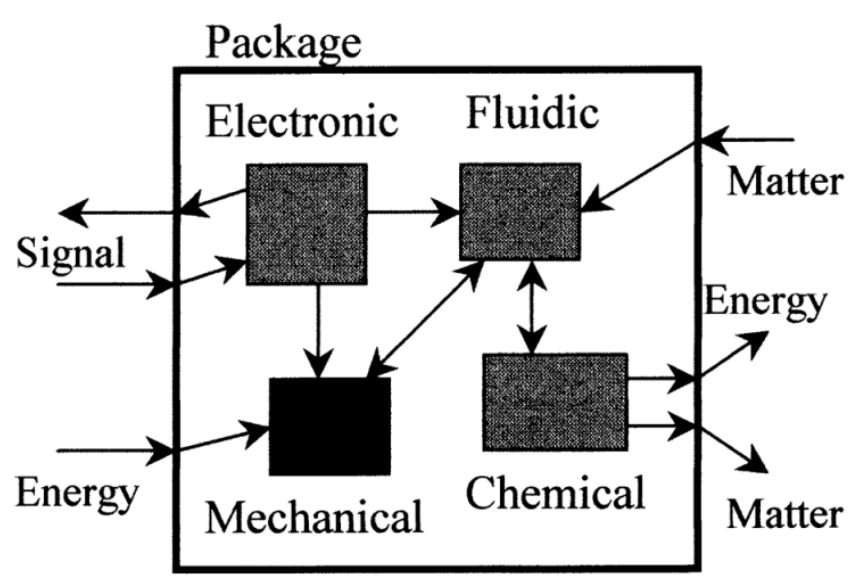

Fig. 2. Representative microsystem. The mechanical subsystem is the focus of this paper.

microelectronic, microoptical, microfluidic, microchemical, etc., or as a hybrid combination (microelectromechanical, etc.). Materials selection enters every aspect of subsystem and microsystem design, with the recognition that a local choice (say, for the fluidic component) can potentially affect other subsystems.

Following Ashby [7], the design process can be divided into three stages: concept, embodiment, and detail (see Fig. 3). In the first stage, the designer requires ranges of values for many classes of materials in order to arrive at the device concept. For instance, it may suffice to know that the Young's modulus of ceramic materials lies in the range 100 to $1000 \mathrm{GPa}$, and that the corresponding range for polymers in 0.01 to $10 \mathrm{GPa}$. Bulk values can typically be used to make this choice. In the next stage, the concept is translated into a class of structures (i.e., embodiment), and the properties of a few materials are required to greater precision (say, 20 to $30 \%$ ) for feasibility analyzes. In this stage, bulk values can be used for many properties within the bounds listed in Table II, although measurements are typically necessary to evaluate residual stresses and intrinsic loss coefficients. In the final phase of design, the designer requires the properties of very few materials to very high precision (typically, 3 to $5 \%$ ) to evaluate the performance and reliability of the device in detail. With a few exceptions, most notably the elastic constants of semiconductor-grade single-crystal silicon, measurements are necessary for all properties of all materials at this stage.

\section{Vi. Materials Selection: The Ashby Method}

In the Ashby approach to materials selection [7], a function is sought to describe the performance $(p)$ of the element under consideration. In general, this function has the form

$$
p=p(F, G, M)
$$

where $\mathrm{F}, \mathrm{G}$ and $\mathrm{M}$ express the functional requirements, geometric parameters, and materials indices, respectively. In many cases, the variables in (9) can be separated to give

$$
p=p_{1}(F) p_{2}(G) p_{3}(M) \text {. }
$$

Equation (10) permits great simplification: For all $\mathrm{F}$ and $\mathrm{G}$, the performance can be optimized by optimizing the appropriate materials indices. This optimization can conveniently be performed using graphs with axes corresponding to different material properties or material indexes. One example of such materials selection charts is the plot of the Young's modulus against density for different materials of interest in MEMS shown in Fig. 4. The following observations can be made from this graph.

i) The scales are chosen to accommodate all the materials of interest. In the context of MEMS, this includes all polymers and elastomers, ceramics and glasses, and metals and alloys.

ii) Materials of a class tend to cluster together; this is true for other charts as well [7]. The two significant exceptions to this observation in Fig. 4 are aluminum and titanium.

iii) Additional useful information can be presented in these graphs. Fig. 4 shows contours of equal wave speed, which plot as a family of parallel lines on this log-log graph.

The selection of materials for micromechanical elements using the concepts of materials indices and selection charts is illustrated in the next section with the aid of several case studies.

\section{CASe Studies}

\section{A. Shock Resistant Microbeams}

Shock survivability is a common requirement for many microsystems. The shock, which can be represented as a pulse of acceleration with amplitude $a$ and duration $\tau$, results in an inertial loading of the structure [33]. If the resonant frequency of the structure is sufficiently high (i.e., the time period of vibration is significantly less than $\tau$ ), the response is quasistatic and the inertial load is given simply by (5). Many MEMS, especially surface-micromachined structures, are susceptible to failure by shock-induced stiction, which occurs when adjacent structures are forced into mechanical contact during the loading event. The higher the bending stiffness, the lower the deflection of the beam, and the lower the probability of stiction. From (8), (5), and (6), the requirements for a shock resistant beam material can be expressed as: i) high $\sqrt{E / \rho}$ to increase the probability of obtaining quasistatic mechanical response, ii) low $\rho$ to decrease the magnitude of the inertial load, and iii) high $\mathrm{E}$ to minimize structural deflection. In other words, we seek materials that maximize the ratio $(E / \rho)$. Fig. 4 can be used to make the initial choice: diamond, silicon carbide, alumina, silicon nitride, and silicon emerge as attractive candidates for fabricating microbeams with high resistance to shock-induced stiction.

Bulk-micromachined MEMS are also susceptible to shockinduced fracture. In addition to low density and high wavespeed, an additional requirement in such structures is a high value of the fracture strength $\left(\sigma_{\mathrm{F}}\right)$. Nominal strength values are plotted against the density for several materials in Fig. 5 . As before, diamond, silicon carbide, alumina, silicon nitride, and silicon emerge as attractive materials for fabricating beams with high resistance to shock-induced fracture. We observe from Fig. 4 that all these materials also exhibit high wave-speeds. 


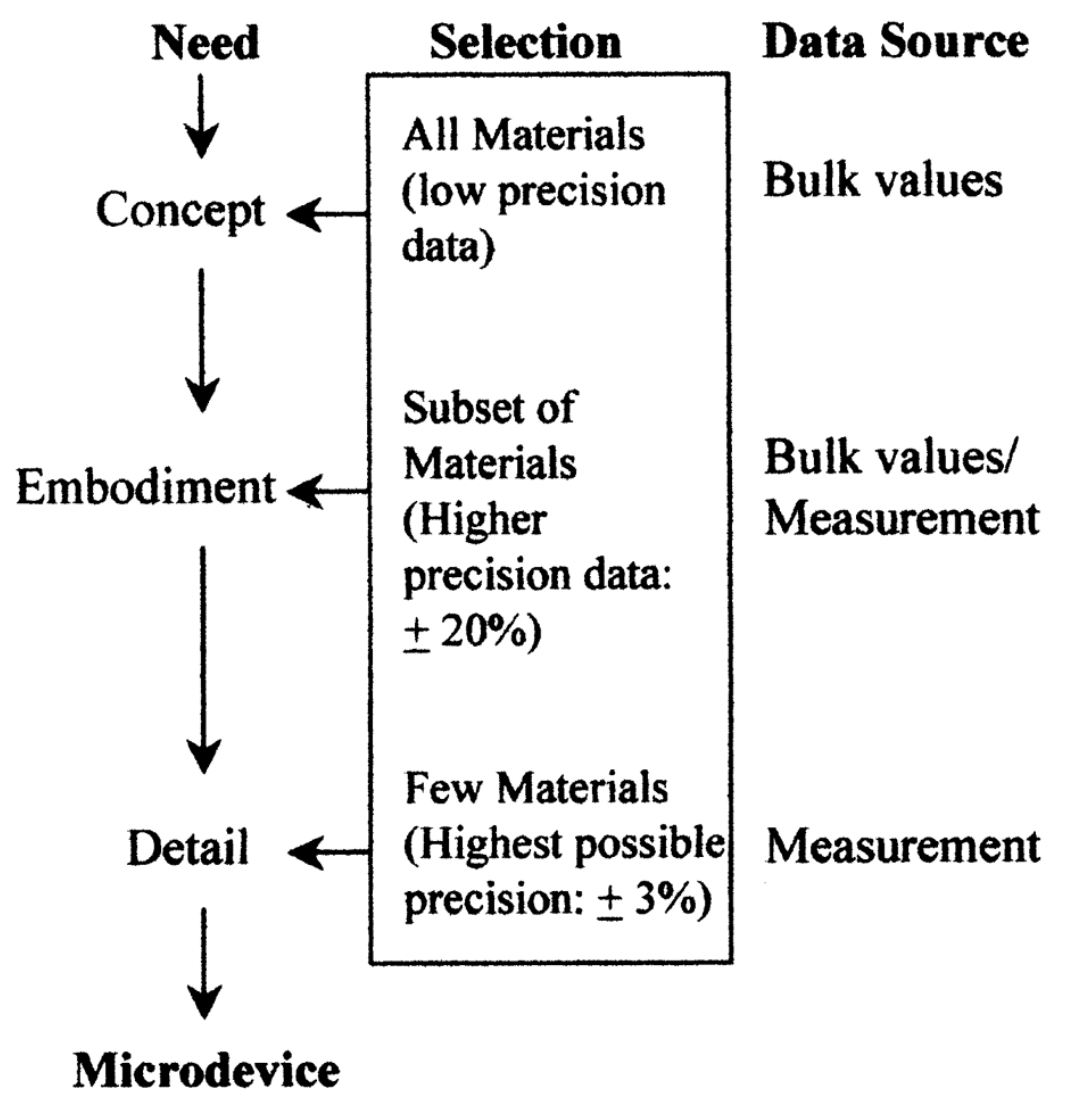

Fig. 3. Schematic illustration of the design process.

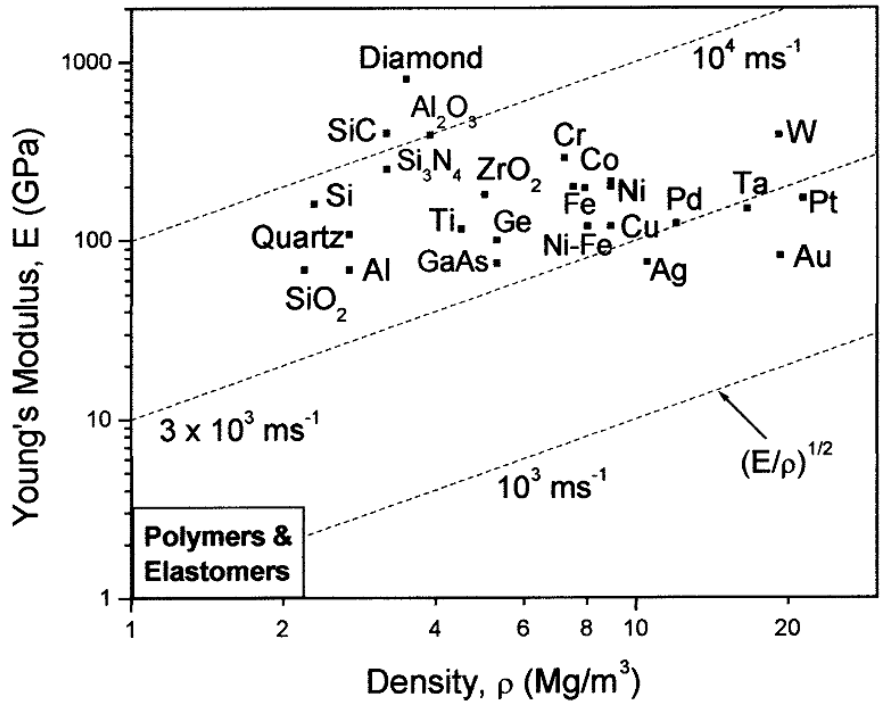

Fig. 4. Young's modulus-density chart for MEMS materials. Contours of constant longitudinal wave velocity $(\sqrt{E / \rho})$ are plotted as parallel lines.

\section{B. Microfabricated Sensors}

A large class of microfabricated sensors measure forces applied to micromachined flexures. Yasumura et al. [27] showed that the sensitivity (i.e., the minimum detectable force, $\mathrm{F}_{\min }$ ) of a cantilever device can be expressed as

$$
F_{\min }=\left(\frac{b h^{2}}{L}\right)^{1 / 2}(k T B)^{1 / 2}\left[\eta^{1 / 2}(E \rho)^{1 / 4}\right]
$$

where $k$ is Boltzmann's constant, $T$ is the absolute temperature, and $B$ is the bandwidth. Equation (11) indicates that the best choice of material for vacuum-operated sensors (with $\eta \approx \eta_{i}$ ) is one that simultaneously minimizes the following materials indexes:

$$
M_{6}=\eta_{i}^{1 / 2} \quad \text { and } \quad M_{7}=(E \rho)^{1 / 4} .
$$

From Fig. 6, the initial choice of materials can be narrowed to silicon dioxide, quartz, silicon, gallium arsenide, and silicon nitride. (Aluminum, which also has a low value of $M_{7}$, exhibits very low intrinsic losses $\left(\sim 10^{-5}\right)$ in bulk form [23]; however, thin film aluminum is typically polycrystalline and the loss coefficient increases by about two orders of magnitude [28].)

Sensors operated under ambient conditions have loss coefficients largely determined by extrinsic losses due to air-damping. The loss coefficient is, to first order, independent of material properties. Therefore, the material of choice is simply one with low values of $(\mathrm{E} \rho)^{1 / 4}$. Fig. 6 suggests that polymers are attractive candidates for such applications. This is consistent with the analysis of Genolet et al. [34], who fabricated a polymeric probe for scanning force microscopy.

\section{Micromechanical Filters}

Micromachined beam resonators are potential candidates for insertion into radio-frequency communications circuits for many frequency-selective applications [35]. Wang et al. [36] identified the requirements for such devices to include a high frequency to match the frequency of the signals of interest 


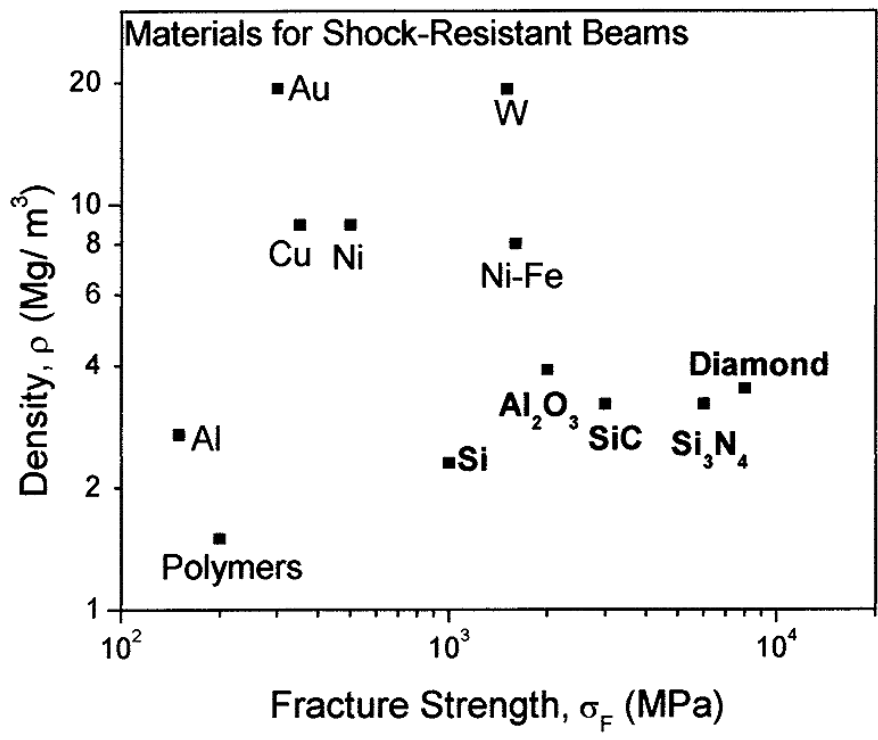

Fig. 5. Density-fracture strength chart for MEMS materials. Materials resistant to shock-induced fracture are those with high strength and low density.

(i.e., large $\sqrt{E / \rho}$ ), a high quality factor to decrease power consumption (i.e., $\eta<10^{-4}$ ), and a high stiffness to increase the dynamic range of capacitively driven resonators (i.e., large E). The initial selection can again be made using Fig. 4. Diamond emerges as the most attractive candidate, as also recognized by Kohn et al. [37] and Wang et al. [38]. (Recent measurements suggest that high quality factors $(>19000)$ can be achieved in chemical-vapor deposited polycrystalline diamond microresonators [38]). The other candidate materials are silicon carbide, alumina, silicon nitride, and silicon.

\section{Micromachined Flexures}

Flexures are integral components of many microsystems. The functional requirements are a large displacement for a small applied force and a large displacement without fracture. From (6) and (7), candidates for flexure materials are those with low values of $E$ and large values of $\left(\sigma_{\mathrm{F}} / \mathrm{E}\right)$. Fig. 7 suggests that polymers are attractive materials for fabricating micromechanical flexures, followed by silicon nitride, Ni-Fe alloys, and silicon.

\section{E. Summary}

The above examples illustrate the use of material indexes and material properties charts. The value of such an exercise lies in the ability to systematically narrow the range of materials under consideration and to identify specific combinations of materials and properties for experimental analysis. This is useful because the measurement of mechanical properties of materials is usually a costly exercise in terms of effort, money, and time.

It is interesting to note that, in the devices considered above, silicon emerges as a good, but not always the best, candidate material. The same conclusion is reached in considering materials for diaphragms in pressure sensors [5], high frequency vibrating elements in gyroscopes [5], rotating disks in power producing micro turbines [5], and high-speed, high-force electrostatic actuators [39].

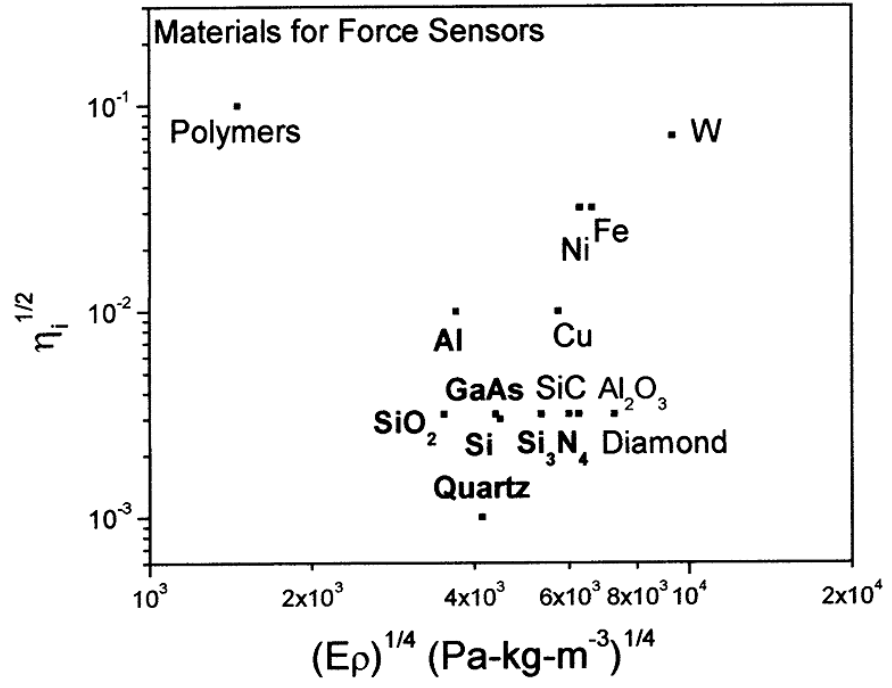

Fig. 6. The square root of intrinsic loss coefficient $\left(\sqrt{\eta_{i}}\right)$ is plotted against $(\sqrt[4]{E \rho})$. Low values of both quantities are required for vacuum-operated force sensors.

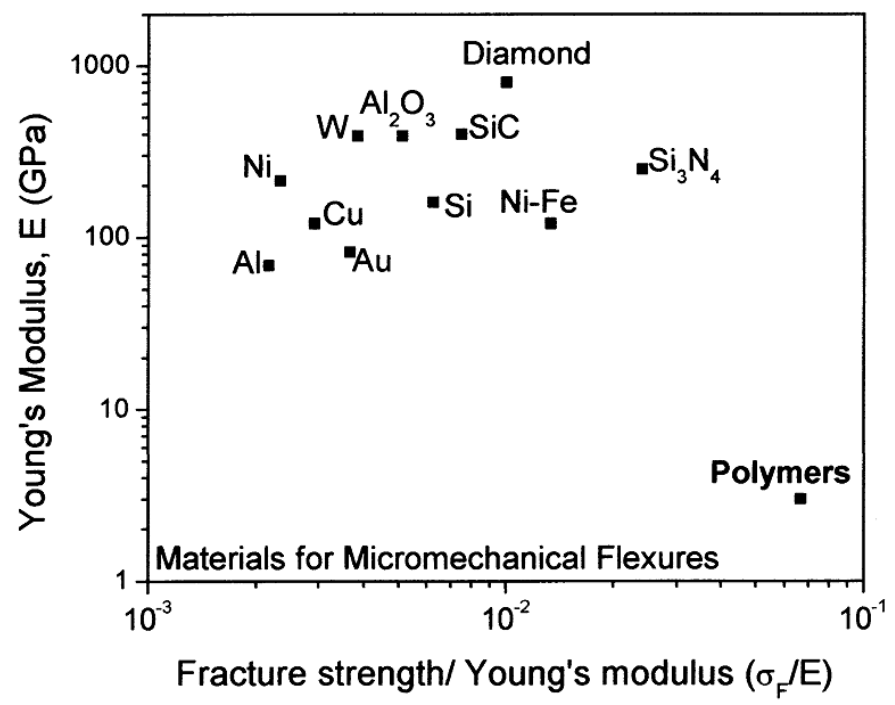

Fig. 7. Micromechanical flexures require a large ratio of the fracture strength to the Young's modulus $\left(\sigma_{\mathrm{F}} / \mathrm{E}\right)$ and a low value of the Young's modulus $(\mathrm{E})$.

\section{FURTHER CONSIDERATIONS}

The concepts and analysis presented in the earlier sections suggest the following areas for further exploration.

\section{A. Materials and Shape}

We have thus far considered mechanical elements with solid prismatic cross-sections. Such shapes dominate microsystems design due to processing constraints. However, it is well established that performance can be enhanced as much, if not more, by changing shapes as by changing materials [7], [10]. For a given area of cross-section, for example, the bending stiffness of a thin-walled tube is much greater than that of a solid section. Materials-shape-performance correlations have been extensively studied in macroscale structures [7], [10], [40]; to the extent that continuum mechanics is valid, these studies apply to micromechanical structures as well. The challenge in using 
these correlations in microsystems design lies primarily in developing new processing techniques for the creation of novel shapes.

\section{B. Multistage Materials Selection}

The single-stage materials selection presented in the case studies can be readily extended to multistage selection. For example, consider the design of a force sensor that will be exposed to shock environments (in space applications, for instance). An approach to selection is to first identify candidate materials for sensors (silicon dioxide, quartz, silicon, gallium arsenide, and silicon nitride) and then, independently, for shock resistance (diamond, silicon carbide, alumina, silicon nitride and silicon), and, finally, to choose from amongst the common members of the two sets (silicon and silicon nitride, in this case). This procedure can be implemented using computer-based methods [41].

\section{Materials Integration}

Microsystems design involves systems-, process-, and materials-integration [1]. As indicated in Fig. 2, the mechanical elements will have to be integrated with the other subsystems. An example is the integration of polymer force sensors, or diamond micromechanical filters, with silicon integrated circuits. Microsystems are invariably composite structures consisting of several materials that are processed using different techniques.

The primary issues associated with materials integration are adhesion, phase stability and reaction, and the origin and evolution of intrinsic and thermomechanical stresses. All these phenomena are the result of complicated interactions involving many different mechanisms; experimental evaluation of specific materials and structures is invariably required at some stage of design. It is worth noting the following.

i) The nucleation of new phases during reactions at the interfaces between compositionally different phases requires interdiffusion [42]. Therefore, even when there is a large thermodynamic driving force for phase formation, reactions can be kinetically constrained by using suitable diffusion barriers.

ii) In some cases, self-limiting phase reactions are desirable because they enhance adhesion. For example, thin aluminum films deposited on oxidized silicon wafers exhibit excellent adhesion because the aluminum reduces silica to form a thin layer of aluminum oxide at the interface; the alumina then serves as a diffusion barrier to prevent further reaction.

iii) The roughness and chemistry of the substrate can significantly influence the evolution of intrinsic stresses in deposited thin films [43]. It is important to account for these effects while measuring intrinsic stresses for design purposes.

iv) It is necessary to consider the entire composite structure, and not merely nearest neighbor interactions, in evaluating thermomechanical stresses. In the limiting case of multiple thin elastic layers on a thick elastic substrate, interactions between adjacent layers, and the details of the stacking sequence, can be neglected. The thermoelastic stresses can then be estimated, to first order, by considering the independent interaction of each film with the substrate [44].

v) The kinetics of activated processes responsible for microstructural evolution, phase transformation, chemical reac- tions, and structural degradation are strongly temperature-dependent. It is possible to prevent, or mitigate, undesirable reactions by appropriate temperature control [1].

\section{CONCLUSION}

The set of materials available to microsystems designers is rapidly expanding. Techniques now exist to introduce and integrate a large number of metals, alloys, ceramics, glasses, polymers, and elastomers into microsystems. As a first step toward an approach for the selection of materials in microsystems design, we considered the initial selection of materials for micromechanical devices with structural dimensions $>1 \mu \mathrm{m}$. The variation of mechanical properties with length scale and processing parameters was considered and bounds for initial design values were suggested. Measurements were identified as being necessary even in the initial stages of design to obtain values for intrinsic stresses and loss coefficients. The concepts of material indexes and material properties charts were reviewed and their utility in materials selection for several representative devices including shock-resistant microbeams, force sensors, micromechanical filters and micromachined flexures were illustrated. Issues associated with the integration of materials into microsystems were briefly discussed.

\section{REFERENCES}

[1] S. D. Senturia, Microsystem Design. Norwell, MA: Kluwer, 2001.

[2] N. I. Maluf, An Introduction to Microelectromechanical Systems Engineering. Norwood, MA: Artech, 2000.

[3] M. J. Madou, Fundamentals of Microfabrication. Boca Raton, FL: CRC, 2002.

[4] N. C. MacDonald, L. Y. Chen, J. J. Yao, Z. L. Zhang, J. A. McMillan, D. C. Thomas, and K. R. Haselton, "Selective chemical vapor deposition of tungsten for microelectromechanical structures," Sens. Actuators, vol. 20, pp. 123-133, 1989.

[5] S. M. Spearing, "Materials issues in microelectromechanical systems (MEMS)," Acta Mater., vol. 48, pp. 179-196, 2000.

[6] - Materials Selection for MEMS, Lecture Notes. Cambridge, MA Massachusetts Institute of Technology, 2002.

[7] M. F. Ashby, Materials Selection in Mechanical Design. Oxford, U.K.: Butterworth-Heinemann, 1999.

[8] — , "On the engineering properties of materials," Acta Metal., vol. 37, pp. 1273-1293, 1989.

[9] M. F. Ashby and D. R. H. Jones, Engineering Materials: An Introduction to their Properties \& Applications. Oxford, U.K.: Butterworth-Heinemann, 1980.

[10] M. F. Ashby, "Materials and shape," Acta Metall. Mater, vol. 39, pp. 1025-1039, 1991.

[11] - "Multi-objective optimization in material design and selection," Acta Mater, vol. 48, pp. 359-369, 2000.

[12] D. Cebon and M. F. Ashby, "Materials selection for precision instruments," Meas. Sci. Technol., vol. 5, pp. 296-306, 1994.

[13] J. E. Huber, N. A. Fleck, and M. F. Ashby, "The selection of mechanical actuators based on performance indices," Proc. R. Soc. Lond. A, vol. 453, pp. 2185-2205, 1997.

[14] J. Shieh, J. E. Huber, N. A. Fleck, and M. F. Ashby, "The selection of sensors," Progr. Mater. Sci., vol. 46, pp. 461-504, 2001.

[15] V. T. Srikar and S. M. Spearing, "A critical review of microscale mechanical testing methods used in the design of microelectromechanical systems (MEMS)," Exper. Mechan., 2003.

[16] W. N. Sharpe, "Mechanical properties of MEMS materials," in The MEMS Handbook, M. Gad-el-Hak, Ed. Boca Raton, FL: CRC Press.

[17] W. D. Nix, "Mechanical properties of thin films," Metall. Trans., vol. 20A, pp. 2217-2245, 1989.

[18] R. E. Mihailovich and N. C. MacDonald, "Dissipation measurements of vacuum-operated single-crystal silicon microresonators," Sens. Actuators, vol. 50A, pp. 199-207, 1995.

[19] C. Zener, Elasticity and Anelasticity of Metals, Illinois: University of Chicago Press, 1948. 
[20] A. S. Nowick and B. S. Berry, Anelastic Relaxation in Crystalline Solids. New York: Academic, 1972.

[21] V. B. Braginsky, V. P. Mitrofanov, and V. I. Panov, Systems with Small Dissipation. Chicago, IL: University of Chicago Press, 1985

[22] B. H. Houston, D. M. Photiadis, M. H. Marcus, J. A. Bucaro, X. Liu, and J. F. Vignola, "Thermoelastic loss in microscale oscillators," Appl. Phys. Lett., vol. 80, pp. 1300-1302, 2002.

[23] V. K. Kinra and K. B. Milligan, "A second-law analysis of thermoelastic damping,” J. Appl. Mech., vol. 61, pp. 71-76, 1994.

[24] R. Lifshitz and M. L. Roukes, "Thermoelastic damping in micro- and nanomechanical systems," Phys. Rev., vol. B61, pp. 5600-5609, 2000.

[25] T. V. Roszhart, "The effect of thermoelastic internal friction on the Q of micromachined silicon resonators," in Tech. Dig. Solid-State Sensor Actuator Workshop, Hilton Head, SC, 1990, pp. 13-16.

[26] V. T. Srikar and S. D. Senturia, "Thermoelastic damping in fine-grained polysilicon flexural beam resonators," J. Microelectromech. Syst., vol. 11, pp. 499-504, 2002.

[27] K. Y. Yasumura, T. D. Stowe, E. M. Chow, T. Pfafman, T. W. Kenny, B. C. Stipe, and D. Rugar, "Quality factors in micron- and submicron- thick cantilevers," J. Microelectromech. Syst., vol. 9, pp. 117-125, 2000.

[28] D. Heinen, H. G. Bohn, and W. Schilling, "Internal friction in freestanding thin Al films," J. Appl. Phys., vol. 78, pp. 893-896, 1995.

[29] C. M. Su, M. Wuttig, A. Fekade, and M. Spencer, "Elastic and anelastic properties of chemical vapor deposited epitaxial 3C-SiC," J. Appl. Phys., vol. 77, pp. 5611-5615, 1995.

[30] M. Ohring, The Materials Science of Thin Films. San Diego, CA: Academic, 1992.

[31] J. A. Floro, E. Chason, R. C. Cammarata, and D. J. Srolovitz, "Physical origins of intrinsic stresses in Volmer-Weber thin films," MRS Bulletin, vol. 27, pp. 19-25, 2002.

[32] C. V. Thompson, Residual Stresses in Thin Films, Lecture notes. Cambridge, MA: Massachusetts Institute of Technology, 2002.

[33] V. T. Srikar and S. D. Senturia, "The reliability of microelectromechanical systems in shock environments," J. Microelectromech. Syst., vol. 11, pp. 206-214, 2002.

[34] G. Genolet, J. Brugger, M. Despont, U. Drechsler, P. Vettiger, N. F. de Rooij, and D. Anselmetti, "Soft, entirely photplastic probes for scanning force microscopy," Rev. Sci. Instrum., vol. 70, pp. 2398-2401, 1999.

[35] J. J. Yao, "RF MEMS from a device perspective," J. Micromech. Microeng., vol. 10, pp. R9-R38, 2000.

[36] K. Wang, A.-C. Wong, and C.T.-C. Nguyen, "VHF free-free beam high-Q micromechanical resonators," J. Microelectromech. Syst., vol. 9, pp. 347-360, 2000.

[37] E. Kohn, M. Adamschik, P. Schmid, S. Ertl, and A. Floter, "Diamond electromechanical micro devices-technology and performance," Diamond and Related Materials, vol. 10, pp. 1684-1691, 2001.

[38] J. Wang, J. E. Butler, D. S. Y. Hsu, and C. T. -C. Nguyen, "High-Q micromechanical resonators in $\mathrm{CH}_{4}$-reactant-optimized high acoustic velocity CVD polydiamond," in Tech. Dig. Solid-State Sensor, Actuator, and Microsystems Workshop, Hilton Head, SC, June 2-6, 2002, pp. 61-62.

[39] V. T. Srikar and S. M. Spearing, "Materials selection for microfabricated electrostatic actuators," Sens. Actuators, vol. 102A, pp. 279-285, 2003.
[40] P. M. Weaver and M. F. Ashby, "Materials limits for shape efficiency," Progr. Mater. Sci., vol. 41, pp. 61-128, 1997.

[41] Cambridge Materials Selector, Granta Design, Cambridge, UK, 1995.

[42] C. V. Thompson, "On the role of diffusion in phase selection during reactions at interfaces," J. Mater. Res., vol. 7, pp. 367-373, 1992.

[43] H. J. Schneeweiß and R. Abermann, "On the influence of the substrate properties on the internal growth stress of titanium films at $250^{\circ} \mathrm{C}$," Thin Solid Films, vol. 228, pp. 40-43, 1993.

[44] P. H. Townsend, D. M. Barnett, and T. A. Brunner, "Elastic relationships in layered composite media with approximation for the case of thin films on a thick substrate," J. Appl. Phys., vol. 62, pp. 4438-4444, 1987.

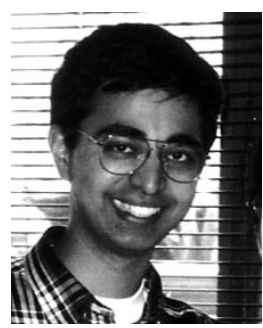

V. T. Srikar received the B.Tech. degree in metallurgical engineering from Banaras Hindu University, Varanasi, India, in 1994 and the Ph.D. degree in materials science from the Massachusetts Institute of Technology (MIT), Cambridge, in 1999 with a dissertation on the electromigration behavior and reliability of submicron aluminum interconnects in silicon integrated circuits.

$\mathrm{He}$ is currently a Postdoctoral Associate at MIT with research interests centered on microscale and nanoscale materials and structures. His recent research activities include studies of the shock reliability of MEMS, identification of a novel mode of thermoelastic damping in polysilicon microresonators, self-assembly of micro- and nanoparticles on the internal surfaces of silicon microsystems, and microscale stress measurement using Raman spectroscopy. $\mathrm{He}$ is involved in the fluidic packaging of the MIT MicroRocket device and in the design and fabrication of a microfabricated solid-oxide fuel cell

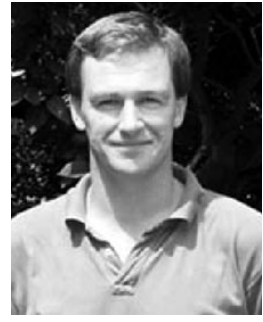

S. Mark Spearing received the Ph.D. degree from Cambridge University Engineering Department, Cambridge, U.K., in 1990.

From 1990 to 1992, he worked as a research engineer at the University of California at Santa Barbara where he produced analytical models for the failure of high-temperature ceramic materials, and at Carborundum Microelectronics, where he was a member of the electronic packaging technical development team, from 1992 to 1994. He is an Associate Professor of Aeronautics and Astronautics at the Massachusetts Institute of Technology (MIT), Cambridge, where he has been since 1994. His technical interests include materials and structural analysis and design of MEMS, electronic packaging and advanced composites. Since 1995, he has been responsible for the materials and structures aspects of the design and packaging of the MIT MicroEngine, MicroRocket, Microchemical Power and Microhydraulic Transducer projects, as well as conducting cross-cutting underpinning technology development.

Dr. Spearing is a Member of the ASME. 